Schweizerisches Jahrbuch für Entwicklungspolitik

27-2 | 2008

Migration und Entwicklung : Eine Zweckallianz

\title{
Migration : eine zu erschliessende Entwicklungsquelle
}

Denis Drechsler and Jason Gagnon

\section{(2) OpenEdition}

12 Journals

Electronic version

URL: http://journals.openedition.org/sjep/317

DOI: $10.4000 /$ sjep.317

ISSN: 1663-9677

Publisher

Institut de hautes études internationales et du développement

Printed version

Date of publication: 1 décembre 2008

Number of pages: 77-95

ISBN: 978-2-940415-08-3

ISSN: 1660-5926

\section{Electronic reference}

Denis Drechsler und Jason Gagnon, « Migration : eine zu erschliessende Entwicklungsquelle », Schweizerisches Jahrbuch für Entwicklungspolitik [Online], 27-2 | 2008, Online erschienen am: 27 Mai 2010, abgerufen am 08 September 2020. URL : http://journals.openedition.org/sjep/317 ; DOI : https://doi.org/10.4000/sjep.317 


\section{Migration: eine zu erschliessende Entwicklungsquelle}

Denis Drechsler und Jason Gagnon*

\section{Einführung}

190 Millionen Menschen leben derzeit nicht in dem Land, in dem sie geboren wurden ${ }^{1}$. Diese Zahl ist in relativem Verhältnis in den letzten vierzig Jahren erstaunlich stabil geblieben und liegt bei rund 3 Prozent der gesamten Weltbevölkerung (siehe Grafik 1). Das Interesse für die Migration und ihre Zusammenhänge mit der Entwicklung nimmt hingegen in allen Teilen der Welt zu - und dies aus mehreren guten Gründen.

Zum einen ist die internationale Wanderungsbewegung in absoluten Zahlen signifikant angestiegen, wobei zu bedenken ist, dass sich die gesamte Weltbevölkerung zwischen 1960 und 2005 mehr als verdoppelt hat. Zweitens ist die Migration, obwohl in den entwickelten Gebieten besonders ausgeprägt, auch in vielen Entwicklungsländern und -gebieten - vor allem als Folge politischer Reformen sowie der Entwicklung der Transport- und Kommunikationsmittel $\mathrm{zu}$ einem wichtigen politischen Thema geworden ${ }^{2}$. Schliesslich tritt die Migration in vielerlei Formen auf (z.B. legale und illegale Migration) und betrifft verschiedene Arbeitsmarktsegmente (z.B. formelle und informelle Arbeitsmärkte); somit ist die Ermittlung der mit der Migration verbundenen Vorteile und Kosten zu einer dringlichen politischen Frage geworden.

Diese Gründe mögen erklären, warum die internationalen Migrationsströme gegenwärtig im Mittelpunkt lebhafter Kontroversen stehen. Während Politiker und Medien häufig auf die mit der Migration verbundenen Kosten - betreffend Sicherheitsfragen oder die Integration von Ausländern - hinweisen, werden die potenziellen Auswirkungen der Migration auf die Entwicklung oft übersehen. Insbesondere die Herkunftsländer sowie die im Lande zurückgebliebenen Angehörigen und Familien der Emigranten können aus der internationalen Migration

* Denis Drechsler, Policy Analyst and Outreach Coordinator, Poverty Reduction and Social Development, OECD Development Centre, OECD, Paris.

Jason Gagnon, Research Associate, Poverty Reduction and Social Development, OECD Development Centre, OECD, Paris.

Die in diesem Artikel geäusserten Meinungen sind jene der Autoren und geben nicht unbedingt die Ansichten der OECD oder ihrer Mitgliedsländer wieder.

1 Diese Zahl umfasst auch 13,5 Millionen Flüchtlinge. Siehe Dilip Ratha and Zhimei Xu, Migration and Remittances Factbook 2008, Washington DC, World Bank, 2008, <http://econ.worldbank.org>

2 So haben beispielsweise die Regierungen Marokkos und der Philippinen ihre Migrationspolitik in zunehmendem Masse als Entwicklungsinstrument eingesetzt. Ein gutes Beispiel einer auf die Migration ausgerichteten internationalen konzertierten Aktion ist die Weltkommission für internationale Migration (World Commission on International Migration, GCIM, <http://www.gcim.org $>$ ) und die sich daran anschliessenden Konferenzen des Weltforums für Migration und Entwicklung (Global Forum for Migration and Development, GFMD) in Brüssel (2007) und in Manila (2008). 
enorme Wohlstandsgewinne erzielen und würden aus einem effizienteren Migrationsmanagement Nutzen ziehen.

Der vorliegende Artikel hat zum Ziel, einen Überblick über die Zusammenhänge zwischen Migration und Entwicklung (Migration-Development Nexus) zu vermitteln. Er zeigt eingangs die neueren Migrationstendenzen auf und stellt die wichtigen Kanäle heraus, über die die internationalen Migrationsströme zur Entwicklung der Herkunftsgebiete beitragen können. Er präsentiert einen Konzeptrahmen zur Analyse der Auswirkungen der Migration auf die Entwicklung den so genannten Migrationszyklus, und befasst sich mit einigen Schwerpunktthemen der Migrationsdebatte - Abwanderung qualifizierter Arbeitskräfte (Braindrain), Verwendung der Migrantenüberweisungen und DiasporaNetzwerke. Der Beitrag schliesst mit konkreten Empfehlungen bezüglich der Politiken zur Maximierung der potenziellen Migrationsgewinne ab.

\section{Grafik 1: Internationale Migranten prozentual zur Weltbevölkerung, 1960-2005}

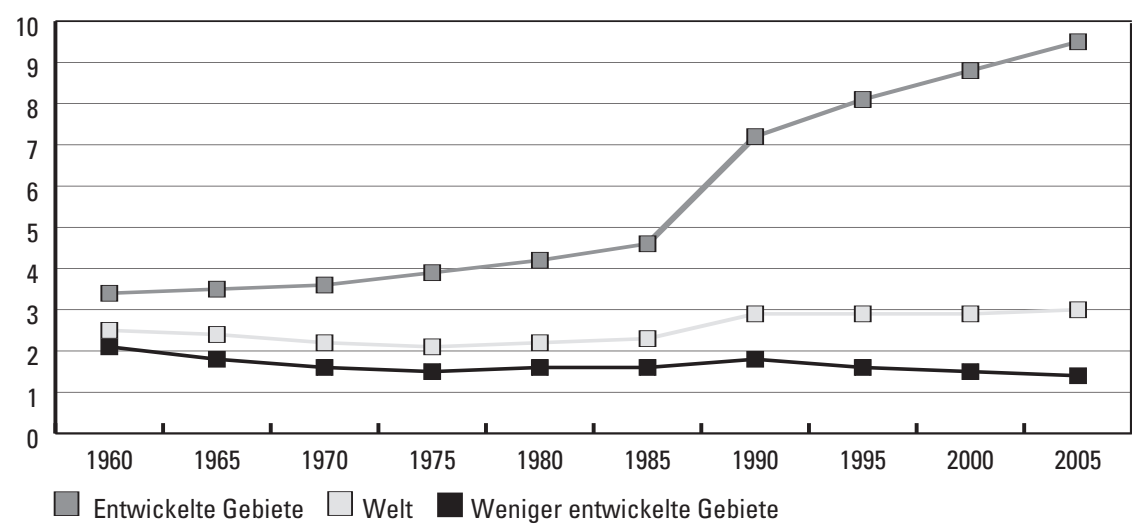

Quelle: Department of Economic and Social Affairs, Population Division, Trends in Total Migrant Stock: The 2005 Revision, POP/DB/MIG/Rev.2005/Doc, New York, United Nations, 2006, <http://esa.un.org/migration>.

Anmerkung: Die „entwickelten Gebiete" umfassen alle Gebiete Europas sowie Nordamerika, Australien, Neuseeland und Japan.

\section{Neuere Migrationstendenzen}

Menschen wandern aus verschiedenen Gründen aus. Zum einen kann die Mobilität erzwungen sein, beispielsweise im Fall eines Konflikts ${ }^{3}$, durch die Migrationspolitik ${ }^{4}$, infolge von Umweltgefahren und technologischen Risiken ${ }^{5}$, Krankheit $^{6}$ oder politischer Vertreibung 7 . In den meisten Fällen beruht die Migration

3 Ruth Haug, ,Forced Migration, Processes of Return and Livelihood Construction among Pastoralists in Northern Sudan“, Disasters, Bd. 26, Nr. 1, 2002, S. 70-84.

4 Jorgen Carling, ,Migration Control and Migrant Fatalities at the Spanish-African Border“, International Migration Review, Bd. 41, Nr. 2, 2007, S. 316-343.

5 Lori Hunter, „Migration and Environmental Hazards“, Population and Environment, Bd. 26, Nr. 4, 2005, S. 273-302.

6 Samuel Clark et al., „Returning Home to Die: Circular Labour Migration and Mortality in South Africa“, Scandinavian Journal of Public Health, Bd. 35, Nr. 3, 2007, S. 35-44.

7 John Salt and J. Stein, „Migration as a Business: The Case of Trafficking“, International Migration, Bd. 35, Nr. 4, 1997, S. 467-494. Ruth Haug, „Forced Migration, Processes of Return and Livelihood Construction among Pastoralists in Northern Sudan“, op. cit. 
aber auf der Entscheidung eines Einzelnen oder einer Familie und gründet sich auf wirtschaftliche Erwägungen.

Nimmt man das Modell von Arthur Lewis zum Ausgangspunkt, so funktionieren die internen und transnationalen Arbeitsmärkte in unvollkommener Weise ${ }^{8}$. Migration ergibt sich aus der Erwartung, andernorts höhere Einnahmen in Form von besseren Löhnen und Gehältern oder einer angemesseneren Vergütung zu erzielen. Somit suchen die Menschen Märkte, wo sie in ihrem Fachbereich oder auf ihrem Präferenzgebiet arbeiten können ${ }^{9}$. Migrationsentscheidungen werden auch im Rahmen eines Haushalts als Mittel zur Bewältigung finanzieller Risiken und zur Diversifizierung der Einkommensquellen getroffen ${ }^{10}$; sie werden mit der Einrichtung von Sozialnetzwerken im Lauf der Zeit bestärkt ${ }^{11}$.

Die traditionellen Faktoren der Migration sind in einem raschen Wandel begriffen, insoweit die Gesamttendenzen mit den Entwicklungen in Bezug auf die bestehenden Zwänge, die Demographie, die Politik, die Wirtschaftsstrukturen und die Familienverhältnisse Schritt halten. Migration wird vermutlich ein grundlegendes Phänomen der heutigen globalisierten Welt bleiben, nicht zuletzt wegen demographischer Faktoren: In den entwickelten Ländern nimmt die Bevölkerung $a b$, wohingegen sie in den Entwicklungsländern zunimmt; dies führt zu einem Überangebot an Arbeitskräften, das den Migrationsdruck erhöht. Die Zwänge ändern sich infolge des technologischen Fortschritts und des gesteigerten Informationsumlaufs, während einige Länder aufgrund der politischen Verhältnisse zunehmend Schwierigkeiten haben, ihre Migrationsströme zu legitimieren. Dies hat zu einem erhöhten Bürokratieaufwand geführt und dazu beigetragen, die Reiserouten der Migranten komplizierter zu machen. Auch hat sich die interne Migration dadurch intensiviert.

Ferner sind zahlreiche weitere Veränderungen der Migrationstendenzen zu beobachten. Zum einen stellt man eine bemerkenswerte Feminisierung der freiwilligen Migration fest: Frauen machen heutzutage 49,6 Prozent der internationalen Migranten aus ${ }^{12}$. Zum anderen wissen wir - vielleicht dank einer besseren Datensammlung - dass die Süd-Süd-Migration dem Umfang nach fast so bedeutend wie die Süd-Nord-Migration ist, und dass sich ein Grossteil davon zwischen angrenzenden Staaten abspielt.

Die allgemeine Verschärfung der Grenzregulierung hat ebenfalls zu Veränderungen im Migrationsverhalten geführt. Migranten zeigen häufig eine Präferenz für zirkuläre Systeme, indem sie zeitweilig zwischen Herkunfs- und Aufnahmegebiet hin und her pendeln. Jedoch können einige Migranten Im Fall einer verschärften Politik beschliessen, im Aufnahmegebiet zu bleiben, da sie befürchten,

8 Arthur Lewis, „Economic Development with Unlimited Supplies of Labour“, Manchester School of Economic and Social Sciences, Bd. 22, Nr. 2, 1954, S. 139-191.

9 Larry A. Sjaastad, „The Costs and Returns of Human Migration“, The Journal of Political Economy, Bd. 70, 1962, S. 80-93. John Harris and M. Todaro, „Migration, Unemployment and Development: A Two-Sector Analysis“, American Economic Review, Bd. 5, Nr. 1, 1970, S. 23-42.

10 Jacob Mincer, „Family Migration Decisions“, Journal of Political Economy, Bd. 86, Nr. 5, 1978, S. 749-773. Oded Stark, The Migration of Labor, Oxford, Basil Blackwell, 1991, 406 S.

11 Paul Winters et al., „Family and Community Networks in Mexico-US Migration“, Journal of Human Resources, Bd. 36, Nr. 1, 2001, S. 159-184. Kaivan Munshi, „Networks in the Modern Economy: Mexican Migrants in the US Labor Market“, Quarterly Journal of Economics, Bd. 118, Nr. 2, 2003, S. 549-597.

12 Dilip Ratha and Zhimei Xu, Migration and Remittances Factbook 2008, World Bank, op. cit. 
nach der Ausreise nicht mehr dorthin zurückkehren zu können. Eine restriktivere Politik hat zudem irreguläre Migrationsbewegungen ausgelöst und, damit einhergehend, den informellen Sektor angekurbelt.

All diese Faktoren zeigen, dass die Migration weiterhin ein wichtiger Bestandteil der globalisierten Welt bleiben wird, und dass sie eine ausgeprägte Wirkung auf die Entwicklungsländer haben wird. Um die potenziellen Vorteile der Migration zu maximieren, ist es daher wesentlich, die Art und Weise, wie die Migration zur Entwicklung beitragen kann, zu verstehen und die mit der Migrationsbewegung verbundenen wirtschaftlichen Herausforderungen zu ermitteln.

\section{Migration und Entwicklung}

\section{Ein Konzeptrahmen: der Migrationszyklus ${ }^{13}$}

Die Migration wirkt sich in komplexer Weise auf die Entwicklung der Herkunftsländer der Migranten aus. Das Modell des Migrationszyklus ist ein nützliches Werkzeug zur Ermittlung der diesbezüglichen Kanäle (siehe Tabelle 1) ${ }^{14}$. Die Migration kann auf dreifache Art das Wachstum ankurbeln und die Armut reduzieren: durch Veränderungen des Arbeitsangebots, durch Veränderungen der Produktivität und durch Geldüberweisungen von Migranten. Der Nettogewinn der Emigration ist die Summe dieser drei Effekte (abzüglich ihrer Kosten).

Das Ausmass eines migrationsbezogenen Schocks auf die Wirtschaft hängt von der Phase des betreffenden Landes im Migrationszyklus ab. Somit kann das Modell Licht auf die Heterogenität der Ergebnisse zwischen verschiedenen Ländern und Regionen werfen. Die fünf Phasen des Migrationszyklus sind: Ausreise, Anpassung, Konsolidierung, Vernetzung und Rückkehr. Jede dieser Phasen geht mit einer variierenden Konfiguration von Schocks und Auswirkungen auf Wachstum und Armut einher. Während die meisten Auswanderungsländer verschiedene Varianten der Phasen des Migrationszyklus durchlaufen, können spezielle Phasen übersprungen oder beschleunigt durchlaufen werden, und die Rückkehr kann mit der Zuwanderung aus anderen Ländern zusammenfallen. Die fünf Phasen sind im Rahmentext 1 kurz zusammengefasst.

Tabelle 1: Ein Modell des Migrationszyklus: seine Auswirkungen auf das Herkunftsland

\begin{tabular}{|c|c|c|c|c|c|}
\hline Phase & Arbeitsangebot & Produktivität & Geldüberweisungen & Wachstum & Armut \\
\hline Ausreise & $\downarrow$ & $\downarrow$ & 0 & $\downarrow$ & $\uparrow$ \\
\hline Anpassung & $\downarrow$ & $?$ & $\uparrow$ & $\downarrow$ & $\uparrow$ \\
\hline Konsolidierung & 0 & $\uparrow$ & 个 & 个 & $\downarrow$ \\
\hline Vernetzung & 0 & $\uparrow$ & $\uparrow$ & $\uparrow$ & $\downarrow$ \\
\hline Rückkehr & $\uparrow$ & $?$ & $\downarrow$ & $?$ & $?$ \\
\hline
\end{tabular}

Quelle: OECD, Policy Coherence for Development: Migration and Developing Countries, Paris, Organisation for Economic Co-operation and Development (OECD), 2007.

Anmerkung: $\uparrow($ Fettdruck $)=($ starke) Steigerung $; \downarrow($ Fettdruck $)=($ starker $)$ Rückgang; $0=$ keine Auswirkung; ? = zweideutige Auswirkung.

13 Dieser Abschnitt ist folgendem Bericht des Entwicklungszentrums der OECD entnommen: Policy Coherence for Development: Migration and Developing Countries, OECD Development Centre, Paris, OECD, 2007.

14 Das Konzept des Migrationszyklus wird auch von Louka Katseli et al. in folgender Publikation beschrieben : Effects of Migration on Sending Countries: What Do We Know?, Working Paper Nr. 250, 


\section{Rahmentext 1: Die fünf Phasen des Migrationszyklus}

\section{Ausreise}

Die erste Phase ist durch die Ausreise der Migranten gekennzeichnet und wirkt sich somit direkt auf das Arbeitskräfteangebot aus. Ob diese Phase zu einem Beschäftigungsrückgang und zu einem Produktionsabfall im Herkunftsland führt, hängt entscheidend vom Grad der Arbeitslosigkeit oder der Unterbeschäftigung ab. Im Fall der Abwanderung hoch qualifizierter Arbeitskräfte ist anzunehmen, dass die Produktivität sinkt. Andererseits kann die Abwanderung wenig qualifizierter Arbeitskräfte zuweilen den Druck auf den Arbeitsmarkt lockern, wenn die Arbeitslosigkeit im Segment der wenig qualifizierten Kräfte hoch war.

\section{Anpassung}

In dem Masse, als die Migration anhält und mehr Migranten das Land verlassen, sinkt die Produktion weiter ab. Jedoch hat eine anhaltende Migration auch zur Folge, die Information über die Beschäftigungsmöglichkeiten in den Zielländern zu verbessern, so dass die mit der Migration verbundenen Informationsbeschaffungs- und Transaktionskosten zurückgehen. Reduzierte Migrationskosten veranlassen ihrerseits die Familienangehörigen oder andere Mitglieder der Gemeinschaft, sich den ursprünglichen Migranten anzuschliessen, und die Haushalte beginnen, die Migration als Strategie zum Verdienst ihres Lebensunterhalts einzusetzen.

\section{Konsolidierung}

Auch wenn die Zusammenführung der Emigrantenfamilien im Zielland anhalten kann, tendieren die internationalen Ströme von Arbeitskräften dazu, sich an einem gegebenen Punkt zu stabilisieren. Die Wirtschaftstätigkeit kann sich in dem Masse verbessern, als die Überweisungen der Migranten zunehmen und die in der Anpassungsphase erfolgte wirtschaftliche Umstrukturierung und Humankapitalanhäufung ihre positiven Wirkungen zeigen. Während dieser Konsolidierungsphase besteht ein Trend zur Wachstumssteigerung und zum Rückgang der Armut. Ein weiteres Absinken der Informationsbeschaffungs- und Verwaltungskosten der Migration führt zu Veränderungen bezüglich der Zusammensetzung und der Charakteristiken der Haushalte im Herkunftsland, deren Mitglieder ausgewandert sind. Die ärmeren Haushalte können es sich leisten, auszuwandern, und ihre Überweisungen an die zurückgebliebenen Familien tragen zur Reduzierung der absoluten Armut und Ungleichheit bei.

\section{Vernetzung}

In der Vernetzungsphase integrieren sich die Migranten besser ins Aufnahmeland und bilden oft Netzwerke transnationaler Gemeinschaften (Diaspora). Die Gründung von "Heimatstadtverbänden“ („hometown associations") im Zielland verbessert die Kommunikation zwischen Herkunfts- und Aufnahmeländern und fördert die Wirtschaftsbeziehungen. Die Migranten senden weiterhin wachstumsfördernde Überweisungen nach Hause, jedoch in geringerem Umfang, besonders wenn ihnen die Familienangehörigen ins Zielland nachgefolgt sind. In der Zwischenzeit hat die Humankapitalanhäufung - die teilweise durch die Überweisungen der Migranten und die sich aus der Auswanderungsmöglichkeit ergebenden opportunistischen Anreize gefördert wurde - zur Heranbildung von Qualifikationen in den Herkunftsländern beigetragen.

\section{Rückkehr}

Wenn die Emigration durch angemessene Politiken zum Entwicklungsprozess, zur Heranbildung von Fachwissen und zu gesteigertem Wachstum im Herkunftsland beitragen konnte, kann sich im betreffenden Land allmählich ein Mangel an Arbeitskräften bemerkbar machen. Die freigewordenen Stellen können durch interne Migration und durch den Zustrom von Arbeitskräften aus den Nachbarländern besetzt werden, jedoch können sie auch die Rückkehr von Auswanderern mit sich bringen. Die Rückwanderer auch jene, die ursprünglich aus ländlichen Gebieten kommen - lassen sich nach der Rückkehr in die Heimat oft in Städten nieder, wodurch sich der Druck auf die städtischen Arbeitsmärkte erhöht. Ob das Wachstum in dieser Phase angekurbelt oder gedämpft wird, hängt davon ab, bis zu welchem Grad die neuen Einwanderer die einheimischen Arbeitskräfte ergänzen oder verdrängen, von den Fachkenntnissen der zurückgekehrten Migranten und von der Flexibilität des Arbeitsmarktes.

Paris, OECD Development Centre, 2006, ferner im OECD-Bericht Policy Coherence for Development: Migration and Developing Countries, OECD Development Centre, op. cit. 
Das Migrationszyklus-Modell bietet einen interessanten und nützlichen Rahmen, um die aktuellen und die erwarteten Migrationstendenzen in den Herkunftsländern, ihre möglichen Auswirkungen und ihre eventuellen politischen Implikationen ersichtlich zu machen. Drei der Komponenten des Migrationszyklus-Modells - die Abwanderung von Arbeitskräften, der Transfer von Geldüberweisungen und die Rückkehr der Migranten (einschliesslich der DiasporaNetzwerke) - verdienen spezielle Beachtung und werden nachstehend untersucht.

\section{Abwanderung von Arbeitskräften und Braindrain}

Die Diskussion um die Auswirkungen der Abwanderung von Arbeitskräften auf die Herkunftsländer der Migranten war bis vor kurzem mit der Debatte über die Emigration Hochqualifizierter, die so genannte „Braindrain-Debatte“ verbunden. Es gibt jedoch mehr Kanäle, über die sich die Emigration auf die lokalen Arbeitsmärkte und die einheimische Wirtschaft auswirken kann, und diese betreffen nicht nur die Abwanderung hoch qualifizierter Personen.

Hieraus lassen sich zwei zentrale politische Ziele ableiten. Zum einen ist die Ermittlung des Qualifikationsniveaus der Emigranten vor der Ausreise wesentlich, um zu verstehen, wie die Migration aus jeder Migrantengruppe den Entwicklungsprozess beeinflusst. Zum anderen können, da sich die Abwanderung hoch qualifizierter Arbeitskräfte abträglich auf das gesamte Humankapital eines Landes auswirken kann, Politiken betreffend die Ab-, Rück- und Weiterwanderung qualifizierter Arbeitskräfte (sog. ,policies of brain circulation“), statt einer Braindrain-Politik, angesprochen werden.

\section{$\square$ Wenig qualifizierte Migranten}

Ein Grossteil der Emigration wenig qualifizierter Arbeitskräfte erfolgt aus Volkswirtschaften, die durch eine geringe Produktivität und ein Überangebot an Arbeitskräften gekennzeichnet sind. Die Abwanderung wenig qualifizierter Arbeitskräfte, auch in grossem Umfang, hat in den meisten Fällen keine starke negative Wirkung auf die einheimische Produktion, sondern trägt statt dessen dazu bei, die Arbeitsmarktverhältnisse für die Zurückgebliebenen zu verbessern.

Die genauen Folgen der Migration wenig qualifizierter Arbeitskräfte auf die Herkunftsländer der Migranten hängt entscheidend vom Beschäftigungsstatus der Migranten vor ihrer Auswanderung ab. Selbst wenn im Herkunftsland hohe Arbeitslosigkeit herrscht, und auch wenn der Migrant arbeitslos war, reduziert die Abwanderung eindeutig das Reservoir von Arbeitslosen.

Insbesondere kann der Abzug wenig qualifizierter Migranten aus den Arbeitsmärkten der Herkunftsländer Möglichkeiten für andere wenig qualifizierte Ersatzarbeiter vor Ort eröffnen. In Ländern mit angespannten Arbeitsmärkten ${ }^{15}$ müssen die Arbeitgeber ihre Lohnangebote erhöhen, um die leer gewordenen Stellen zu besetzen. Die Gesamtproduktion geht - zumindest kurzfristig zurück, und die Arbeitgeber können von den Lohnerhöhungen betroffen werden.

15 Ein angespannter Arbeitsmarkt ist durch kurze und seltene Perioden der Arbeitslosigkeit und durch geringe Unterbeschäftigung gekennzeichnet (C. Pissarides, ,Search Unemployment with On-the-job Search", Review of Economic Studies, Bd. 61, Nr. 3, 1994, S. 457-475). 
Die wenig qualifizierten Arbeitskräfte profitieren von den höheren Löhnen, die sie jetzt verlangen können, und mit der Zeit passen sich die verschiedenen Produktionstätigkeiten dem zurückgegangenen Arbeitsangebot an wenig qualifizierten Kräften an, und die Produktion steigt erneut. Im Gegensatz hierzu können die Emigranten in Ländern mit einer hohen Anzahl an Arbeitslosen ohne weiteres mit geringem Aufwand oder ohne Kosten für die Arbeitgeber ersetzt werden. Die den Arbeitgebern auferlegten Kosten sind in diesem Fall definitionsgemäss minimal und die Gesamtproduktion wird kaum beeinträchtigt. Für die im Lande verbliebenen ungelernten Arbeiter stellt dies einen Gewinn dar, da sie rascher Arbeit finden, oder indem sie ihre beruflichen Aufstiegschancen wahrnehmen und in bessere Positionen vorrücken.

\section{$\square$ Hoch qualifizierte Migranten}

Die Abwanderung hoch qualifizierter Arbeitskräfte, der so genannte „Braindrain“, wird weitgehend als einer der negativsten Aspekte der internationalen Migration angesehen. Das gesamte Ausmass und die Verbreitung des internationalen Braindrains ist stark umstritten, und bis vor kurzem hatten Politiker und Forscher mangels systematischer Datenquellen lediglich eine Ahnung von der Grössenordnung des Phänomens.

Die Karibik, Zentralamerika, die Inseln des Pazifik und Teile von Afrika sind verhältnismässig die am stärksten betroffenen Gebiete (siehe Karte 1); in absoluten Zahlen jedoch dominiert die Abwanderung von chinesischen, indischen und brasilianischen Qualifizierten ${ }^{16}$. Der Braindrain stellt ein wesentliches Problem auf dem afrikanischen Kontinent dar, da zehn der 53 afrikanischen Länder eine tertiäre Auswanderungsquote ${ }^{17}$ von über 35 Prozent aufweisen. Die kleinen Länder sind mit einer Auswanderungsquote von Hochqualifizierten von rund 30 Prozent, ${ }^{18}$ und bei einigen Ländern (Surinam, Guyana, Jamaika und Haiti) sogar von über 80 Prozent, ${ }^{19}$ besonders anfällig.

Die Determinanten des Braindrains sind vielfältig. Wenn man den Braindrain analysiert, so wird bei der Ermittlung des Ausmasses der Abwanderung hoch qualifizierter Arbeitskräfte im Allgemeinen zuerst das Niveau der auf den Qualifikationen beruhenden, positiven Selbstauswahl berücksichtigt. Wie von Mark Rosenzweig bzw. von Jeffrey Grogger und Gordon Hanson dargelegt, sind der Hauptfaktor der Migrationsentscheidung eher die Vergütungen der Qualifikationen (rewards to skills) als die Löhne allein ${ }^{20}$.

16 Frédéric Docquier and H. Rapoport, Skilled Migration: The Perspective of Developing Countries, IZA Discussion Papers Nr. 2873, Bonn, Institute for the Study of Labor (IZA), 2007. Frédéric Docquier et al., Brain Drain in Developing Countries, Working Paper Nr. 4, Louvain-la-Neuve, Département des sciences économiques de l'Université catholique de Louvain, 2007.

17 Abdeslam Marfouk definiert die tertiäre Emigration als Abwanderung von Personen, deren Ausbildung über die Oberschule (high school) hinausreicht (Abdeslam Marfouk, The African Brain Drain: Scope and Determinants, Working Papers DULBEA Nr. 08-07.RS, Bruxelles, Université libre de Bruxelles, Department of Applied Economics, 2008).

18 Michel Beine et al., Brain Drain and Its Determinants: A Major Issue for Small States, IZA Discussion Paper Nr. 3398, Bonn, Institute for the Study of Labor (IZA), 2008.

19 Dilip Ratha and Zhimei Xu, Migration and Remittances Factbook 2008, World Bank, op. cit.

20 Mark Rosenzweig „The Global Migration of Skill“, presentation made at the Migration and Development Conference in Lille, France, June 2008. Jeffrey Grogger and G.H. Hanson, Income Maximization and the Selection and Sorting of International Migrants, NBER Working Papers Nr. 13821, Cambridge MA, National Bureau of Economic Research, Inc., 2008. 


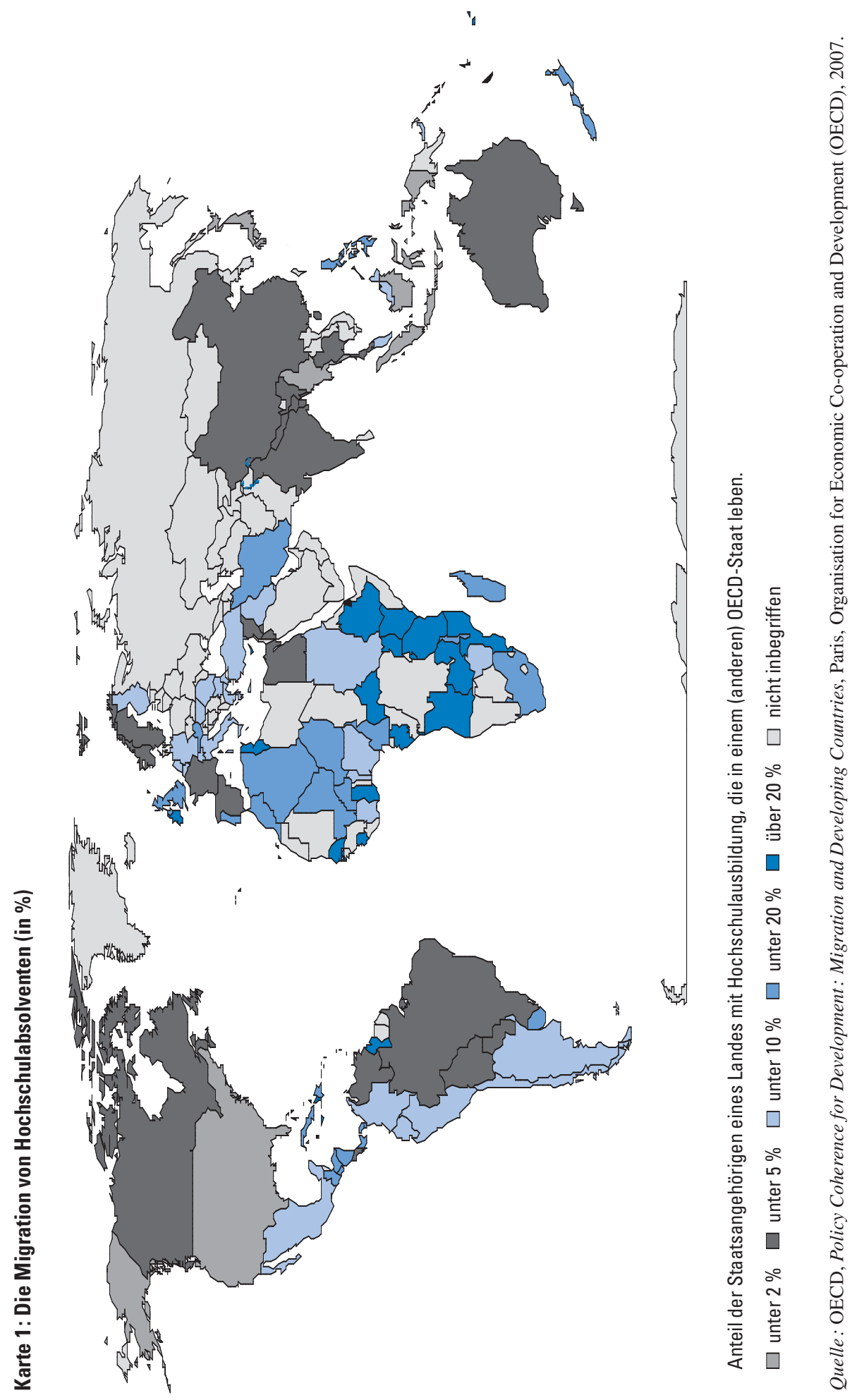


Die Zunahme der Emigration Hochqualifizierter hat aber auch noch zwei weitere klare Determinanten. Zum einen haben sich die sich verschlechternden Arbeitsbedingungen und der unzureichende Kreditzugang im Vergleich zu den Zielländern als wichtige Push-Faktoren für Einzelne und Familien erwiesen, die ihren Lebensstandard verbessern wollen. Zum anderen haben die beim Informationsumlauf seit kurzem eingetretenen Fortschritte den potenziellen Emigranten wirksame Instrumente an die Hand gegeben, um ihre Auswanderung besser vorzubereiten und umzusetzen. Diese Verbesserungen sind auf die Informationstechnologie zurückzuführen, aber auch auf die sozialen Netzwerke, die von der Diaspora im Laufe der Zeit errichtet werden. Weitere identifizierte Faktoren, die einen starken Einfluss auf die Abwanderung Hochqualifizierter haben, sind der Aufgeschlossenheitsgrad des Herkunftslandes, die Rückständigkeit seines internen Schulssystems, seine relative Grösse, seine Kolonialbeziehungen sowie der Grad an politischer Instabilität im Herkunftsland ${ }^{21}$, oder auch die Unterschiede zwischen Aufnahmeland und Herkunftsland in Bezug auf das Gesundheitswesen und die medizinische Infrastruktur ${ }^{22}$.

Die negativen Auswirkungen des Braindrains sind oft mit dem Wachstum bzw. mit einem Mangel an Wachstum verbunden. Dies ist zumindest aus drei Gründen wesentlich. Der erste Grund ist ein direkter Arbeitsmarkteffekt, bei dem das gesamte Humankapital in der Wirtschaft in dem Masse abnimmt, als die hoch qualifizierten Arbeitskräfte abwandern, mit einem eindeutigen Produktivitätsrückgang. Der zweite Grund ist ein Verlust an Kapital durch Steuerausgaben für die Ausbildung. Wird die Ausbildung aus öffentlichen Mitteln finanziert, so muss der Staat seine Beiträge erhöhen, um den Humankapitalverlust auszugleichen. Mit der Abwanderung hoch qualifizierter Arbeitskräfte ändert sich zudem das Verhältnis zwischen ungelernten und gelernten Arbeitern, was zu höheren Löhnen für die Hochqualifizierten im Lande führt und wiederum mehr Ungleichheit nach sich ziehen kann. Auch ist die Abwanderung hoch qualifizierter Arbeitskräfte mit einer Einbusse an positiven externen Effekten und Nebenwirkungen für die Zurückgebliebenen verbunden, wenn die Emigranten die Beziehungen zur Wirtschaft ihres Herkunftslandes abbrechen.

\section{$\square$ Vom „Braindrain“ zur „Brain Circulation“}

Die Abwanderung hochqualifizierter Kräfte muss für die Herkunftsländer nicht unbedingt von Nachteil sein. Unter gewissen Umständen kann sie sogar vom Verlust („Braindrain“) zum Gewinn („Braingain“) werden. Wenn beispielsweise bezüglich der Migration keine Sicherheit besteht, kann der Braindrain die Produktivität im Herkunftsland steigern, indem er einen Anreiz für Humankapitalinvestitionen bietet ${ }^{23}$. Michel Beine et al. zeigen, dass zwei unterscheidbare Effekte mit dem Braindrain verbunden sind: Einerseits gibt es einen „brain effect", insofern als die Migrationsaussichten die potenziellen Migranten veranlassen, in ihr Humankapital zu investieren. Andererseits stellt der ",drain effect “ den Verlust dar, der mit der tatsächlichen Abwanderung dieser ausgebildeten

21 Frédéric Docquier et al., Brain Drain in Developing Countries, op. cit.

22 Alok Bhargava and F. Docquier, „HIV Pandemic, Medical Brain Drain, and Economic Development in Sub-Saharan Africa“, The World Bank Economic Review, Bd. 22, Nr. 2, 2008, S. 345-366.

23 Andrew Mountford, „Can a Brain Drain Be Good for Growth in the Source Economy?“, Journal of Development Economics, Bd. 53, Nr. 2, 1997, S. 287-303. Jean-Pierre Vidal, ,The Effect of Emigration on Human Capital Formation“, Journal of Population Economics, Bd. 11, Nr. 4, 1998, S. 589-600. 
Migranten aus ihrem Land verbunden is $\mathrm{t}^{24}$. Überwiegt der „,brain effect“ über den „drain effect“, dann besteht die Möglichkeit eines so genannten „positiven Braindrains" (,beneficial brain drain“). Michel Beine et al. zeigen auf, dass dies bis zu einem bestimmten Punkt bei der Abwanderung Hochqualifizierter der Fall ist, über den hinaus die mit dem Braindrain verbundenen Wachstumseffekte negativ $\operatorname{sind}^{25}$. Die Forschung weist nach, dass sich die Braingain-Theorien in gewissen Fällen bestätigen ${ }^{26}$.

Effiziente Politiken zur Behandlung der Frage der Migration Hochqualifizierter festzulegen und umzusetzen, ist keine einfache Sache. Ein Grossteil der Braindrain-Debatte ist als Reaktion darauf entbrannt, dass die länderspezifischen Einwanderungsgesetze aufgrund des, ,internationalen Wettbewerbs um die besten Köpfe" zunehmend selektiver werden. Die Debatte veranlasste den Ökonom Jagdish Bhagwati, die Besteuerung hoch qualifizierter Migranten mit einer Zusatzabgabe vorzuschlagen, die als Ausgleich an das Herkunftsland zurückzuzahlen wäre. Obwohl die sog. Bhagwati-Steuer schliesslich aus politischen und logistischen Gründen abgelehnt wurde, ist sie heute weiterhin Diskussionsgegenstand.

Mit dem Aufkommen neuer Braingain-Theorien und positiver externer Ergebnisse im Zusammenhang mit dem Braindrain sind die Politiker vor einiger Zeit vom Versuch abgekommen, die Migrationsströme hoch qualifizierter Arbeitskräfte zu verlangsamen, und sind dazu übergegangen, die Ab-, Rück- und Weiterwanderung hoch qualifizierter Migranten (brain circulation) durch zirkuläre Migrationssysteme zu steigern. Durch die Förderung der zirkulären Migration bieten die Politiker den Migranten den Anreiz, auf zweifache Art direkt zur Wirtschaftsentwicklung ihrer Herkunftsländer beizutragen : indem sie Gelder in ihre Heimat überweisen, und indem sie mit wertvoller Berufserfahrung in ihr Land zurückkehren ${ }^{27}$.

\section{Überweisungen}

Die Geldtransfers der Migranten in ihr Herkunftsland sind ein wichtiger Faktor und stellen eine wesentliche Verbindung zwischen Migration und Entwicklung dar. Bis vor kurzem war sehr wenig über ihren Umfang bekannt, doch gibt es heutzutage genauere Schätzungen der Migrantenüberweisungen, auch wenn ihr absoluter Umfang wahrscheinlich viel grösser ist.

\section{$\square$ Umfang der Überweisungen}

Die Rücküberweisungen in die Herkunftsländer und die Geldtransfers aus diesen Ländern haben sich mit der Zeit ständig erhöht. Für 2007 waren Rücküberwei-

24 Michel Beine et al., „Brain Drain and Economic Growth: Theory and Evidence“, Journal of Development Economics, Bd. 64, Nr. 1, 2001, S. 275-289.

25 Michel Beine et al., Brain Drain and LDCs' Growth: Winners and Losers, IZA Discussion Paper Nr. 819, Bonn, Institute for the Study of Labor (IZA), 2003.

26 Catia Batista et al., Brain Drain or Brain Gain? Micro Evidence from an African Success Story, IZA Discussion Paper Nr. 3035, Bonn, Institute for the Study of Labor (IZA), 2007.

27 Karin Mayr and G. Peri, Return Migration as Channel of Brain Gain, CReAM Discussion Paper Series Nr. 04/08, London, Centre for Research and Analysis of Migration (CReAM), Department of Economics, University College London, 2008. 
sungen in Höhe von 318 Milliarden Dollar veranschlagt, davon ging ein geschätzter Betrag von 240 Millarden Dollar (75\%) in die Entwicklungsländer ${ }^{28}$. Auch wenn diese Zahlen wegen der uneinheitlichen Datenqualität ${ }^{29}$ mit grosser Vorsicht $\mathrm{zu}$ interpretieren sind, rivalisieren sie mit jenen der öffentlichen Entwicklungshilfe (Official Development Aid, ODA) und der ausländischen Direktinvestitionen (Foreign Direct Investments, FDI) in Ländern mit schwachem und mittlerem Einkommen ${ }^{30}$.

Mehrere Faktoren beeinflussen den Betrag der Überweisungen, einschliesslich die Migrationsdauer. Migranten, die sich lange im Ausland aufgehalten haben, werden vermutlich höhere Beträge überweisen, da ihr Einkommen generell mit der im Aufnahmeland verbrachten Zeit ansteigt. Andererseits kann längere Abwesenheit dazu betragen, das Engagement gegenüber den im Lande verbliebenen Angehörigen zu verringern. Zudem lassen Migranten mit längerer Aufenthaltsdauer oftmals ihre Familien in das Aufnahmeland nachkommen und schicken dann weniger Geld in ihr Heimatland.

Ein weiterer - aber damit verbundener - Faktor, der sich auf die Überweisungen auswirkt, ist die Rückkehrabsicht eines Migranten, die mit seinem Qualifikationsniveau zusammenhängen kann. Im Allgemeinen erfolgt die Migration weniger qualifzierter Arbeitskräfte aus den ärmeren Bevölkerungsschichten. Wenn diese Migranten beabsichtigen, in ihr Land zurückzukehren, und vor allem, wenn ihre engsten Familienmitglieder im Herkunftsland verblieben sind, können die Rücküberweisungen sehr hoch sein. So sind die Überweisungen pro Migrant im Persischen Golf - wo die Migranten wenig qualifiziert sind und nicht von ihren Familienangehörigen begleitet werden - bei weitem die höchsten der Welt ${ }^{31}$. Qualifizierte Migranten neigen ihrerseits dazu, weniger Geld zu überweisen als ihre weniger qualifizierten Kollegen ${ }^{32}$. Somit kann man in gewisser Hinsicht sagen, dass die wenig qualifizierten Migranten überdurchschnittlich zur Entwicklung des Herkunftslandes und zum Rückgang der Armut beitragen. Die Frage, ob die Überweisungen der Migranten aus den ärmsten Ländern den Humankapitalverlust in angemessener Weise ausgleichen - besonders im Segment der hoch qualifizierten Arbeitskräfte (z.B. das afrikanische medizinische Personal) - ist Gegenstand lebhafter Debatten.

28 Dilip Ratha and Zhimei Xu, Migration and Remittances Factbook 2008, World Bank, op. cit.

29 Caroline Freund and N. Spatafora, Remittances: Transaction Costs, Determinants and Informal Flows, Policy Research Working Paper Series Nr. 3704, Washington DC, World Bank, 2005.

30 Lucas, R.E.B., International Migration and Economic Development: Lessons from Low-Income Countries, Cheltenham, UK, Edward Elgar, 2005. UN Department of Economic and Social Affairs, Population Division, International Migration and Development Fact Sheet, New York, United Nations, 2006. Dilip Ratha and Zhimei Xu, Migration and Remittances Factbook 2008, World Bank, op. cit.

Für eine systematische Übersicht über die Geldüberweisungen im Kontext von Subsahara-Afrika siehe: Flore Gubert, „L'impact des transferts de fonds sur le développement des pays d'origine: le cas de 1'Afrique“, in OCDE (dir.), Migration, transferts et développement, Paris, OCDE, 2005, S. 43-72. Die vielleicht umfassendste Analyse der Auswirkungen der Überweisungen ist die Studie von Pablo Acosta et al. zu Lateinamerika: ,What is the Impact of International Remittances on Poverty and Inequality in Latin America ?", World Development, Bd. 36, Nr. 1, 2008, S. 89-114.

31 OECD, Policy Coherence for Development: Migration and Developing Countries, op. cit.

32 Yoko Niimi et al., Remittances and the Brain Drain: Skilled Migrants Do Remit Less, IZA Discussion Paper Nr. 3393, Bonn, Institute for the Study of Labor (IZA), 2008. 


\section{$\square$ Verwendung der Überweisungen}

Auch wenn die Wirkung der Überweisungen vom jeweiligen Kontext abhängen kann, so ist jetzt ganz gut nachgewiesen, dass sich die Überweisungen armutsmindernd auf die Haushalte auswirken, denen sie zukommen, wie etwa in Ghana $^{33}$. Auf den Philippinen zeigen Claudia Martinez und Dean Yang beispielsweise, dass die positiven Wechselkursschwankungen im Aufnahmeland - und somit der Wert der überwiesenen Gelder - das Armutsniveau der Haushalte, aus denen Migranten abgewandert sind, reduzieren ${ }^{34}$. Bontch-Osmolowski et al. kommen ihrerseits zum Ergebnis, dass ein Fünftel der Armutsminderung in Nepal zwischen 1995 und 2004 den Rücküberweisungen zugeschrieben werden kann ${ }^{35}$.

Eine weitere sehr wichtige Debatte betreffend die Überweisungen dreht sich um die Frage, ob diese für produktive Zwecke eingesetzt werden oder lediglich einem erhöhten Konsum dienen. Hierbei sollte jedoch bedacht werden, dass gewisse Formen reinen Konsums für arme Familien als produktive Investitionen angesehen werden können. Zum Beispiel kann der Kauf eines Eisschranks mit dem aus dem Ausland überwiesenen Geld eine produktive Anlage sein, welche die Gesundheit und damit das Humankapital verbessert und potenziell als Grundlage für ein Mikrounternehmen dient ${ }^{36}$. Jedoch ist die Steigerung der Entwicklungseffekte der Überweisungen durch die Ausrichtung der Gelder auf produktivere Zwecke eine wichtige politische Option, die in einigen Ländern bereits zum Einsatz kommt, wie beispielsweise das „Tres per uno"-Programm in Mexiko.

Neben der Debatte über die Verwendung der Überweisungen ist auch die Verteilung der überwiesenen Gelder innerhalb des Haushalts wichtig. Dabei geht es um die Frage, ob die Gelder hauptsächlich von den Erwachsenen verbraucht werden, oder ob sie auch den Kindern für bessere Gesundheit und Ausbildung zugute kommen. Man hat in der Tat festgestellt, dass die Überweisungen in gewissen Fällen die Ausgaben für Schule und Bildung erhöhen können ${ }^{37}$; jedoch war diese Auswirkung in anderen Fällen weniger klar oder sogar negativ ${ }^{38}$. In Gebieten mit grossen Familien und heterogenen Verwandschaftsbeziehungen kann die Verwendung der überwiesenen Gelder eventuell nicht gleichmässig aufgeteilt werden, sondern gemäss einer spezifischen Hierarchie erfolgen.

33 Richard H. Adams Jr, Remittances and Poverty in Ghana, Policy Research Working Paper Series, Nr. 3838, Washington DC, World Bank, 2006.

34 Claudia Martinez and D. Yang, ,Remittances and Poverty in Migrants' Home Areas: Evidence from the Philippines“, in Caglar Ozden and Maurice Schiff (eds.), International Migration, Remittances, and the Brain Drain, Washington DC, World Bank, 2005.

35 Mikhail Bontch-Osmolovski et al., Work-related Migration and Poverty Reduction in Nepal, Policy Research Working Paper Series, Nr. 4231, Washington DC, World Bank, 2007.

36 Für weitere Angaben in diesem Zusammenhang siehe: OECD, Policy Coherence for Development: Migration and Developing Countries, op. cit.

37 Siehe zum Beispiel für die Philippinen: Claudia Martinez and D. Yang, „,Remittances and Poverty in Migrants' Home Areas: Evidence from the Philippines“, op. cit.; für El Salvador: Alejandra Cox Edwards and M. Ureta, International Migration, Remittances, and Schooling: Evidence from El Salvador, NBER Working Papers Nr. 9766, Cambridge, MA, National Bureau of Economic Research Inc., 2003; und für Ecuador: Carla Calero et al., Remittances, Liquidity Constraints and Human Capital Investments in Ecuador, Working Papers - General Series, Nr. 458, The Hague, Institute of Social Studies, 2008.

38 David McKenzie and H. Rapoport, Can Migration Reduce Educational Attainment? Evidence from Mexico, World Bank Policy Research Working Paper Nr. 3952, Washington DC, World Bank, 2006. 
Ausser einer direkten Auswirkung auf die Empfänger der überwiesenen Gelder kommen die Überweisungen auch der lokalen Gemeinschaft zugute. Zum Beispiel kann das ausgegebene Geld Einkommen für die Lieferanten der gekauften Waren und die Erbringer der Dienstleistungen schaffen, die diese Einkünfte wiederum ausgeben und damit eine Kettenreaktion auslösen (Multiplikatoreffekte). Zudem können die in Kleinunternehmen investierten Mittel direkt zur Schaffung von Arbeitsplätzen auf Gemeinschaftsebene dienen ${ }^{39}$.

\section{Rückkehr der Migranten und Diaspora-Netzwerke}

Die Rückkehr der Migranten kann mehrere Formen annehmen, einschliesslich der endgültigen Rückwanderung, der saisonbedingten Migration und der temporären Migration. Die endgültige Rückwanderung (wenn die Migranten mit der Absicht in ihr Heimatland zurückkehren, sich dort dauerhaft wieder niederzulassen) kann gewollt oder erzwungen, geplant oder ungeplant sein. Die Folge ist in jedem Fall, dass die Migranten sich wieder in einem Umfeld integrieren müssen, in dem sie einst gelebt haben, aber mit neuen Aussichten und mit den im Ausland erworbenen wertvollen Ersparnissen. Die Rückkehrer können auch quasi dauerhafte Gebietsansässige sein, die ihr Land systematisch in saisonbedingten ${ }^{40}$ oder temporären Abständen zur Arbeit verlassen, um danach wieder mit den gewünschten Ersparnissen in ihr Herkunftsland zurückzukehren. Zudem kann die Migration zirkulär sein, wenn sowohl die Auswanderung als auch die Rückwanderung temporär sind. Migranten dieser Art siedeln sich niemals dauerhaft an, sondern wählen eine transnationale Existenz, indem sie zwischen zwei (oder mehr) Gebieten hin und her pendeln.

Die Rückkehr kann der lokalen Entwicklung zugute kommen. Migranten, die im besten arbeitsfähigen Alter in die Heimat zurückkehren, tragen oft zur wirtschaftlichen Entwicklung ihres Landes bei, indem sie Kenntnisse und ökonomische Nebenwirkungen generieren. Eine wachsende empirische Literatur verweist auf die Tatsache, dass die zurückkehrenden Migranten Tendenz haben, zu Unternehmern zu werden, wobei sie Mikrounternehmen gründen, die im Ausland erworbenen Fertigkeiten und (finanziellen) Ressourcen einsetzen sowie lokales Personal zur Bewirtschaftung des Betriebs einstellen ${ }^{41}$. Die Neben-

39 Louka Katseli et al., Effects of Migration on Sending Countries: What Do We Know?, op. cit.; OECD, Policy Coherence for Development: Migration and Developing Countries, op. cit.

40 Es handelt sich hier um Aufenthalte von unter einem Jahr; alle anderen Arten von Verträgen mit Aufenthalten von über einem Jahr werden als temporär bezeichnet. Sowohl die saisonbedingte als auch die temporäre Migration kann zirkulär sein, wenn die gleiche Person die betreffenden Grenzen oder Gebiete mehrmals überquert.

41 Nadeed Ilahi, ,Return Migration and Occupational Change“, Review of Development Economics, Bd. 3, Nr. 2, 1999, S. 170-186. Barry McCormick and J. Wahba, „Overseas Work Experience, Savings and Entrepreneurship amongst Return Migrants to LDCs“, Scottish Journal of Political Economy, Bd. 48, Nr. 2, 2001, S. 164-178. Richard Black et al., Migration, Return and Small Enterprise Development in Ghana: A Route out of Poverty?, Sussex Migration Working Paper Nr. 9, Brighton, University of Sussex, 2003. Alice Mesnard, „Temporary Migration and Self-Employment: Evidence from Tunisia“, Brussels Economic Review, Bd. 47, Nr. 1, 2004, S. 119-138. Talip Kilic et al., Investing Back Home: Return Migration and Business Ownership in Albania, Policy Research Working Paper Nr. 4366, Washington DC, World Bank, 2007. Gil Epstein and D.C. Radu, „Returns to Return Migration and Determinants of Subsequent Moves“, EALE Conference Paper, EALE Annual Conference, 20-22 September, Oslo, 2007. Benjamin Aleman-Castilla, The Returns to Temporary Migration to the United States: Evidence from the Mexican Urban Employment Survey, CEP Discussion Papers Nr. dp0804, London, Centre for Economic Performance, LSE, 2007. 
wirkungen können sich auch in Form von sozialem oder Humankapital niederschlagen $^{42}$. Nichtsdestoweniger war es für mehrere politische Institutionen eine schwierige Aufgabe, einen ausgeglichenen Ansatz $\mathrm{zu}$ finden, um produktive Migranten zur Rückkehr zu verleiten, sie wieder in ihrem Heimatland zu integrieren und zum Bleiben zu bewegen.

Die Migranten können sich auch an der Wirtschaft ihres Heimatlandes beteiligen, ohne zurückzukehren, indem sie sich in virtuellen Formen der Rückkehr engagieren. Die Diaspora-Netzwerke stellen ein äusserst wirksames Entwicklungsinstrument dar. Sie tragen nicht nur direkt zur Wirtschaft des Herkunftslandes bei, indem sie Überweisungen in die Heimat senden und den Handel zwischen Aufnahme- und Herkunftsgebieten erleichtern, sondern dienen auch als Informationskanäle. Transnationale Netzwerke geben das Sozial- und Wirtschaftsleben von Gemeinschaften über nationale Grenzen hinweg wieder und verbinden die Migranten mit ihren im Lande verbliebenen Angehörigen. Sie können die Form von „Heimatstadtverbänden“ (,hometown associations“) annehmen und eine Vielzahl von Zielen haben (z.B. Investitionen in der Heimatstadt, Unterstützung der Integration). Migranten unterhalten oft enge Beziehungen zu ihrem Heimatland, auch wenn sie im neuen Gebiet integriert sind. Die Erforschung des Migrationsphänomens wird durch die Vielfalt transnationaler Existenzen erschwert; eine besondere Schwierigkeit liegt darin, festzustellen, wo und wann der Begriff „Nation" anfängt, und wann er aufhört ${ }^{43}$.

\section{Empfehlungen für eine angemessene Politik: Synergien nutzen}

Die Migration kann auf verschiedene Art und Weise zum Entwicklungsprozess in den Herkunftsländern beitragen. Um die sich aus der internationalen Arbeitsmobilität ergebenden Vorteile zu maximieren, müssen aber sowohl die Herkunftsländer als auch die Aufnahmeländer die richtige Politik verfolgen. Vor allem müssen die Länder erkennen, dass die Migration ein transversaler Prozess ist, der sich auf viele Politikbereiche - insbesondere auf die Entwicklung - auswirkt.

Verschiedene politische Instrumente - die Migrations- und die Entwicklungshilfepolitik, aber auch die Handelspolitik - sind miteinander verbunden, und die dadurch ausgelösten Ströme - Migranten, Überweisungen, Entwicklungshilfeaufwendungen, Aus- und Einfuhren - hängen ebenfalls miteinander zusammen. Durch eine sorgfältige Analyse dieser Politiken, durch die Nutzung von Synergien und durch Vermeiden des Aufkommens von sog. „coherence orphans “ das heisst von Gebieten, die Hilfe erhalten, aber aus keinem der anderen Ströme Nutzen ziehen - können die Entwicklungsziele wirksamer erreicht werden, was nachdrücklich für eine kohärentere Beschlussfassung unter den entsprechenden politischen Gemeinschaften plädiert. Somit weist die Migrationspolitik mehrere Facetten auf, und ein verbessertes Migrationsmanagement erfordert die Einrichtung nachhaltiger Partnerschaften zwischen Herkunfts- und Aufnahmegebieten.

42 Maria Enchautegui, ,The Value of US Labor Market Experience in the Home Country: The Case of Puerto Rican Return Migrants“, Economic Development and Cultural Change, Bd. 42, Nr. 1, 1993, S. 169-191.

43 Nina Glick Schiller and A. Wimmer, „Methodological Nationalism, the Social Sciences, and the Study of Migration: An Essay in Historical Epistemology“, The International Migration Review, Bd. 37, Nr. 3, 2003, S. 576-610. 


\section{Aufnahmeländer}

Die Aufnahmeländer der Migranten müssen bei der Gestaltung der Migrationspolitik die möglichen Auswirkungen auf die Entwicklung der Herkunftsländer berücksichtigen. Die Migration und die Entwicklungszusammenarbeit müssen zur Erreichung all ihrer Ziele besser miteinander verbunden werden. Kohärentere Politiken können dazu beitragen, einen potenziellen Humankapitalverlust (Braindrain) in einen Humankapitalgewinn (Braingain) oder in eine zirkuläre Migration hoch qualifizierter Arbeitskräfte (brain circulation) zu verwandeln und die Vorteile der Mobilität wenig qualifizierter Arbeitskräfte für die Herkunftsgebiete zu vermehren. Diese Politiken müssen das Potenzial der zirkulären Migration nutzen und sich gleichzeitig den Herausforderungen der irregulären Migration stellen.

Somit lassen sich die Empfehlungen betreffend die Rolle der Aufnahmegebiete der Migranten wie folgt zusammenfassen :

1. Innovative Kreislaufsysteme (gefördert durch Visa mit mehrfacher Einreiseerlaubnis und Benutzung, durch Arbeitsgenehmigungen oder durch den Abbau interner Migrationsbeschränkungen) können zur einer besseren Steuerung der Migrationsströme beitragen. Im Fall der hoch qualifzierten Migranten können solche Systeme die nachteiligen Auswirkungen auf die Sozialdienste der Herkunftsländer abfedern. Für die wenig qualifizierten Migranten können die Kreislaufsysteme die Überweisungen fördern und die Verbreitung der irreguären Migration reduzieren.

2. Die OECD-Staaten und/oder die industrialisierten Gebiete sollten Leitlinien für die Rekrutierung hoch qualifizierter Arbeitskräfte ausarbeiten und die möglichen negativen Auswirkungen berücksichtigen, die ihre Rekrutierung auf die Entwicklung der Herkunftsgebiete haben kann.

3. Die OECD-Staaten und/oder die industrialisierten Gebiete sollten gemeinsame Massnahmen ergreifen, um die Kosten der Geldtransfers über die offiziellen Kanäle zu senken. Zusätzlich sollten Banken und Finanzinstitute, in Zusammenarbeit mit Finanzinstituten in den Entwicklungsgebieten, die Federführung bei der Ausdehnung der Finanzdienstleistungen auf die armen ländlichen Gebiete übernehmen.

4. Ein Partnerschaftsansatz sollte die Migrationspolitiken der OECD-Staaten und/oder der industrialisierten Gebiete und die Politiken zur Entwicklung der menschlichen Ressourcen der Nicht-OECD-Länder und/oder der ländlichen Gebiete sowie ihre Arbeitsmarkt- und Sozialpolitiken verbinden.

\section{Herkunftsländer}

Die Herkunftsländer der Migranten werden aufgefordert, eine Migrationsdimension in ihre nationale Entwicklungspolitik zu integrieren. Diese Länder müssen ihre Regulierungen überarbeiten und sich fragen. ob ihre makroökonomische Politik, ihre Verwaltung der menschlichen Ressourcen, ihre Hochschulausbildung, ihre Infrastrukturinvestitionen und ihre „Süd-Süd“- oder ,ländlich-ländlich“Initiativen auf regionaler Ebene die Anpassung und den Wiedereinsatz ihrer menschlichen Ressourcen angesichts der zunehmenden Mobilität erleichtern. Die 
Herkunftsländer sollten die Zusammenarbeit mit den Diaspora-Netzwerken fördern und sowohl die Migranten als auch die Rückkehrer bei der Festlegung von co-development-Politiken konsultieren. Initiativen sollten gewährleisten, dass die hoch qualifizierten Fachkräfte zurückkehren, dass die Rückkehrer daraus Nutzen ziehen, dass sie erwerbstätig sind und an der sozialen und wirtschaftlichen Entwicklung ihrer Gemeinschaften teilhaben.

Ferner lassen sich die Empfehlungen für die Integration der Migration in die Entwicklungsstrategien wie folgt zusammenfassen:

1. Die grossen Emigrationsgebiete sollten zahlreiche Aspekte ihrer makroökonmischen Politik - einschliesslich Steuern, Ausgaben und Wechselkursraten - der Abwanderung der Arbeitskräfte anpassen.

2. Die Herkunftsgebiete müssen ihre Arbeitskräftepotenzial-Politik im öffentlichen wie im privaten Sektor der Abwanderung anpassen, um die Angleichung und Auffüllung des Personalbestands zu erleichtern; eine solche Politik sollte zumindest nicht Migranten benachteiligen, die zurückkehren und sich wieder in den Arbeitsmarkt eingliedern wollen.

3. Die Finanzierung der Hochschulausbildung, einschliesslich der finanziellen Unterstützung bedürftiger Studenten und der Unterrichtsplanung, muss die Möglichkeit erwägen, dass einige, ja sogar viele Studierende abwandern können.

4. Beschlüsse betreffend die Infrastrukturinvestitionen müssen Durchgangswege zur Gewährleistung der Mobilität (mobility corridors) berücksichtigen; verbesserte Transport- und Kommunikationskapazitäten können zwischenzeitlich dazu beitragen, die Arbeitsmärkte der Abwanderung anzupassen.

5. Regionale Initiativen unter den Entwicklungsländern müssen mit der Unterstützung der OECD-Staaten und/oder der industrialisierten Gebiete gestärkt werden; ein Grossteil der Migration wenig qualifizierter Arbeitskräfte aus den ärmsten Regionen erfolgt oft in andere nahe gelegene Entwicklungsgebiete.

Ein grösseres Mass an Kohärenz - zwischen den Ministerien innerhalb eines Landes oder Gebiets und unter den Ländern oder Gebieten - zwischen der Migrationspolitik, der Politik der Entwicklungszusammenarbeit sowie der Beschäftigungs-, Handels- und Sicherheitspolitik ist erforderlich, damit alle Beteiligten mehr Nutzen aus der Migration ziehen. Die bestehenden institutionellen Systeme müssen überprüft werden, um die Politikkohärenz zu erhöhen; die Regionen müssen nach Mechanismen suchen, um die Kommunikation, Verhandlung und Konsensbildung unter den politischen Gemeinschaften und ihren Wählerschaften zu fördern. Die OECD-Staaten und/oder die industrialisierten Gebiete müssen die Migrations- und die Entwicklungspolitiken miteinander verbinden; die Entwicklungsgebiete müssen die Migration ihrerseits zu einem Schwerpunkt ihrer nationalen Entwicklungsstrategien machen. Verstärkte Partnerschaften zwischen den Aufnahme- und den Herkunftsländern können ein effizienter Mechanismus ein, um zu gewährleisten, dass miteinander verbundene und kohärente Politiken erarbeitet und angemessen umgesetzt werden. Ferner müssen Fragen betreffend Migration, Beschäftigung, Handel, Investitionen und Entwicklungshilfe gemeinsam auf regionaler, nationaler und globaler Ebene angegangen werden. 
Wie der Verkehr von Gütern und Dienstleistungen ist auch die Migration ein kennzeichnendes Merkmal der Globalisierung. In Anbetracht der sinkenden Kosten der Mobilität, der rasch anwachsenden Bevölkerung in einigen Ländern und einer alternden Bevölkerung in anderen Ländern, und angesichts der zunehmenden Möglichkeiten, am internationalen Mobilitätsprozess im Verlauf der Entwicklung teilzuhaben, kann die internationale Migration mittel- bis langfristig nur noch mehr an Bedeutung gewinnen.

Daher sind Diskussionen über Möglichkeiten, der Migration Einhalt zu gebieten, irreführed und fruchtlos. Stattdessen sollten die Länder vielmehr versuchen, durch angemessene Politiken den potenziellen Nutzen der Migration für alle beteiligten Akteure - Herkunftsländer und Aufnahmeländer der Migranten sowie die Migranten selbst - zu maximieren.

Dieser Artikel hat die bedeutenden Vorteile hervorgehoben, die den Herkunftsländern der Migranten aus dem aufkommenden internationalen Mobilitätssystem erwachsen können. Um nur einige der Herausforderungen zu nennen: Der mit der Migration verbundene Humankapitalverlust (Braindrain) muss in einen Humankapitalgewinn (Braingain) oder in ein zirkuläres Migrationssystem hochqualifizierter Arbeitskräfte (brain circulation) umgewandelt werden. Die Auswirkungen der Überweisungen der Migranten auf den Entwicklungsprozess müssen verbessert werden, indem man die Gelder produktiveren Verwendungszwecken zuführt. Schliesslich sollten die Politiker die Diaspora-Netzwerke viel stärker mit einbeziehen, als dies gegenwärtig der Fall ist.

Die Migration ist nicht ohne Risiken für die Herkunftsländer. Richtig angegangen kann sie aber Möglichkeiten und Vorteile bieten, die ihre eventuellen Kosten bei weitem aufwiegen können. Lassen Sie uns deshalb das der Migration innewohnende, unerschlossene Entwicklungspotenzial anerkennen.

\section{Bibliographie}

Acosta, Pablo, C. Calderon, P. Fajnzylber and H. Lopez, „What is the Impact of International Remittances on Poverty and Inequality in Latin America?", World Development, Bd. 36, Nr. 1, 2008, S. 89-114.

Adams, Richard H. Jr, Remittances and Poverty in Ghana, Policy Research Working Paper Series, Nr. 3838, Washington DC, World Bank, 2006.

Aleman-Castilla, Benjamin, The Returns to Temporary Migration to the United States: Evidence from the Mexican Urban Employment Survey, CEP Discussion Papers Nr. dp0804, London, Centre for Economic Performance, LSE, 2007.

Batista, Catia, A. Lacuesta and P.C. Vicente, Brain Drain or Brain Gain? Micro Evidence from an African Success Story, IZA Discussion Paper Nr. 3035, Bonn, Institute for the Study of Labor (IZA), 2007.

Beine, Michel, F. Docquier and H. Rapoport, „Brain Drain and Economic Growth: Theory and Evidence“, Journal of Development Economics, Bd. 64, Nr. 1, 2001, S. 275-289.

Beine, Michel, F. Docquier and H. Rapoport, Brain Drain and LDCs' Growth: Winners and Losers, IZA Discussion Paper Nr. 819, Bonn, Institute for the Study of Labor (IZA), 2003.

Beine, Michel, F. Docquier and M. Schiff, Brain Drain and Its Determinants: A Major Issue for Small States, IZA Discussion Paper Nr. 3398, Bonn, Institute for the Study of Labor (IZA), 2008.

Bhargava, Alok and F. Docquier, „HIV Pandemic, Medical Brain Drain, and Economic Development in Sub-Saharan Africa“, The World Bank Economic Review, Bd. 22, Nr. 2, 2008, S. 345-366. 
Black, Richard, R. King and R. Tiemoko, Migration, Return and Small Enterprise Development in Ghana: A Route out of Poverty?, Sussex Migration Working Paper Nr. 9, Brighton, University of Sussex, 2003.

Bontch-Osmolovski, Mikhail, E. Glinskaya and M. Lokshin, Work-related Migration and Poverty Reduction in Nepal, Policy Research Working Paper Series Nr. 4231, Washington DC, World Bank, 2007.

Calero, Carla, A.S. Bedi and R. Sparrow, Remittances, Liquidity Constraints and Human Capital Investments in Ecuador, Working Papers - General Series Nr. 458, The Hague, Institute of Social Studies, 2008.

Carling, Jorgen, „Migration Control and Migrant Fatalities at the Spanish-African Border“, International Migration Review, Bd. 41, Nr. 2, 2007, S. 316-343.

Clark, Samuel, M. Collinson, K. Kahn, K. Drullinger and S. Tollman, „Returning Home to Die: Circular Labour Migration and Mortality in South Africa“, Scandinavian Journal of Public Health, Bd. 35, Nr. 3, 2007, S. 35-44.

Docquier, Frédéric and H. Rapoport, Skilled Migration: The Perspective of Developing Countries, IZA Discussion Papers Nr. 2873, Bonn, Institute for the Study of Labor (IZA), 2007.

Docquier, Frédéric, O. Lohest and A. Marfouk, Brain Drain in Developing Countries, Working Paper Nr. 4, Louvain-la-Neuve, Département des sciences économiques de l'Université catholique de Louvain, 2007.

Edwards, Alejandra Cox and M. Ureta, International Migration, Remittances, and Schooling: Evidence from El Salvador, NBER Working Papers Nr. 9766, Cambridge, MA, National Bureau of Economic Research, Inc., 2003.

Epstein, Gil and D.C. Radu, „Returns to Return Migration and Determinants of Subsequent Moves“, EALE Conference Paper, EALE Annual Conference, 20-22 September, Oslo, 2007.

Enchautegui, Maria, „The Value of US Labor Market Experience in the Home Country: The Case of Puerto Rican Return Migrants“, Economic Development and Cultural Change, Bd. 42, Nr. 1, 1993, S. 169-191.

Freund, Caroline and N. Spatafora, Remittances: Transaction Costs, Determinants and Informal Flows, Policy Research Working Paper Series Nr. 3704, Washington DC, World Bank, 2005.

Glick Schiller, Nina and A. Wimmer, ,Methodological Nationalism, the Social Sciences, and the Study of Migration: An Essay in Historical Epistemology“, The International Migration Review, Bd. 37, Nr. 3, 2003, S. 576-610.

Grogger, Jeffrey and G.H. Hanson, Income Maximization and the Selection and Sorting of International Migrants, NBER Working Papers Nr. 13821, Cambridge, MA, National Bureau of Economic Research, Inc., 2008.

Harris, John and M. Todaro, „Migration, Unemployment and Development: A Two-Sector Analysis“, American Economic Review, Bd. 5, Nr. 1, 1970, S. 23-42.

Haug, Ruth, „Forced Migration, Processes of Return and Livelihood Construction among Pastoralists in Northern Sudan“, Disasters, Bd. 26, Nr. 1, 2002, S. 70-84.

Hunter, Lori, „Migration and Environmental Hazards“, Population and Environment, Bd. 26, Nr. 4, 2005, S. 273-302.

Ilahi, Nadeed, „Return Migration and Occupational Change“, Review of Development Economics, Bd. 3, Nr. 2, 1999, S. 170-186.

Katseli, Louka, R.E.B. Lucas and T. Xenogiani, Effects of Migration on Sending Countries : What Do We Know?, Working Paper Nr. 250, Paris, OECD Development Centre, 2006.

Kilic, Talip, C. Carletto, B. Davis and A. Zezza, Investing Back Home: Return Migration and Business Ownership in Albania, Policy Research Working Paper Nr. 4366, Washington DC, World Bank, 2007.

Lewis, Arthur, „Economic Development with Unlimited Supplies of Labour“, Manchester School of Economic and Social Sciences, Bd. 22, Nr. 2, 1954, S. 139-191.

Lucas, R.E.B., International Migration and Economic Development: Lessons from Low-Income Countries, Cheltenham, UK, Edward Elgar, 2005.

Marfouk, Abdeslam, The African Brain Drain: Scope and Determinants, Working Papers DULBEA Nr. 08-07.RS, Bruxelles, Université libre de Bruxelles, Department of Applied Economics, 2008.

Martinez, Claudia and D. Yang, ,Remittances and Poverty in Migrants’ Home Areas: Evidence from the Philippines“, in Caglar Ozden and Maurice Schiff (eds.), International Migration, Remittances, and the Brain Drain, Washington DC, World Bank, 2005.

Mayr, Karin and G. Peri, Return Migration as Channel of Brain Gain, CReAM Discussion Paper Series Nr. 04/08, London, Centre for Research and Analysis of Migration (CReAM), Department of Economics, University College London, 2008.

McCormick, Barry and J. Wahba, „Overseas Work Experience, Savings and Entrepreneurship amongst Return Migrants to LDCs“, Scottish Journal of Political Economy, Bd. 48, Nr. 2, 2001, S. 164-178. 
McKenzie, David and H. Rapoport, Can Migration Reduce Educational Attainment? Evidence from Mexico, World Bank Policy Research Working Paper Nr. 3952, Washington DC, World Bank, 2006.

Mesnard, Alice, „Temporary Migration and Self-Employment: Evidence from Tunisia“, Brussels Economic Review, Bd. 47, Nr. 1, 2004, S. 119-138.

Mincer, Jacob, „Family Migration Decisions“, Journal of Political Economy, Bd. 86, Nr. 5, 1978, S. 749-773.

Mountford, Andrew, „Can a Brain Drain Be Good for Growth in the Source Economy?“, Journal of Development Economics, Bd. 53, Nr. 2, 1997, S. 287-303.

Munshi, Kaivan, „Networks in the Modern Economy: Mexican Migrants in the US Labor Market“, Quarterly Journal of Economics, Bd. 118, Nr. 2, 2003, S. 549-597.

Niimi, Yoko, C. Ozden and M. Schiff, Remittances and the Brain Drain: Skilled Migrants Do Remit Less, IZA Discussion Paper Nr. 3393, Bonn, Institute for the Study of Labor (IZA), 2008.

OCDE, Migration, transferts de fonds et développement, Paris, Organisation de coopération et de développement économiques (OCDE), 2005.

OECD, Policy Coherence for Development: Migration and Developing Countries, Paris, Organisation for Economic Co-operation and Development (OECD), 2007.

Pissarides, C., „Search Unemployment with on-the-job Search“, Review of Economic Studies, Bd. 61, Nr. 3, 1994, S. 457-475.

Ratha, Dilip and Zhimei Xu, Migration and Remittances Factbook 2008, Washington DC, World Bank, 2008.

Rosenzweig, Mark, „The Global Migration of Skill“, presentation made at the Migration and Development Conference in Lille, France, June 2008.

Salt, John and J. Stein, „Migration as a Business: The Case of Trafficking“, International Migration, Bd. 35, Nr. 4, 1997, S. 467-494.

Sjaastad, Larry A., „The Costs and Returns of Human Migration“, The Journal of Political Economy, Bd. 70, 1962, S. 80-93.

Stark, Oded, The Migration of Labor, Oxford, Basil Blackwell, 1991, 406 S.

UN Department of Economic and Social Affairs, Population Division, International Migration and Development Fact Sheet, New York, United Nations, 2006.

UN Department of Economic and Social Affairs, Population Division, Trends in Total Migrant Stock: The 2005 Revision, POP/DB/MIG/Rev.2005/Doc, New York, United Nations, 2006, $<$ http://esa.un.org/ migration $>$.

Vidal, Jean-Pierre, „The Effect of Emigration on Human Capital Formation“, Journal of Population Economics, Bd. 11, Nr. 4, 1998, S. 589-600.

Winters, Paul, A. de Janvry and E. Sadoulet, „Family and Community Networks in Mexico-US Migration“, Journal of Human Resources, Bd. 36, Nr. 1, 2001, S. 159-184. 